\title{
Assessment of CASP gene polymorphisms in periodontal disease
}

\author{
S.W. Kang ${ }^{1 *}$, S.K. Kim ${ }^{1 *}$, J.H. Chung ${ }^{1}$ and J.Y. Ban ${ }^{2,3}$ \\ ${ }^{1}$ Kohwang Medical Institute, School of Medicine, Kyung Hee University, \\ Seoul, Korea \\ 2Department of Dental Pharmacology, School of Dentistry, \\ Dankook University, Cheonan, Korea \\ ${ }^{3}$ Department of Medical Laser, Graduate School, \\ Dankook University, Cheonan, Korea \\ *These authors contributed equally to this study. \\ Corresponding author: J.Y. Ban \\ E-mail: jyban@dankook.ac.kr \\ Genet. Mol. Res. 14 (4): 18069-18077 (2015) \\ Received August b10, 2015 \\ Accepted October 7, 2015 \\ Published December 22, 2015 \\ DOI http://dx.doi.org/10.4238/2015.December.22.33
}

ABSTRACT. Caspases (CASP) are intracellular proteases that play roles as mediators of apoptosis. Activation of caspase 3 is enhanced in chronic periodontitis. Thus, we hypothesized that single nucleotide polymorphisms (SNPs) of CASP genes might be associated with this condition in the Korean population. To investigate whether such polymorphisms might be involved in the development of periodontal disease, 51 patients and 33 control subjects were assessed. A total of 201 CASP gene SNPs were analyzed with genotypes being determined using and Axiom ${ }^{\mathrm{TM}}$ genomewide human assay. SNPStats and SPSS 18.0 were used for the analysis of genetic data and logistic regression models were utilized to evaluate odds ratios, 95\% confidence intervals, and $\mathrm{P}$ values. Of the 201 SNPs, only three (rs12108497, rs4647602, and rs113420705, all in the CASP3 gene) were significantly associated with chronic periodontitis $(P<0.05)$. The minor allele frequencies of these SNPs were higher in the patient group than in the control group. In addition, the TC and GT haplotypes 
formed by rs4647602 and rs113420705 were found to be associated with chronic this disease (TC haplotype, $\mathrm{P}=0.0039$; GT haplotype, $\mathrm{P}$ $=0.002$ ). These results suggest that CASP3 gene polymorphisms may be associated with susceptibility to periodontal disease in the Korean population.

Key words: Association study; Periodontal disease; CASP genes; Polymorphism

\section{INTRODUCTION}

Tooth loss is highly associated with quality of life (Gerritsen et al., 2010). Periodontitis, a major cause of tooth loss, is common among adults in all regions and is found at a rate of 5-15\% in most populations (Burt et al., 1990; Petersen, 2003). Between 2009 and 2012, 46\% of US adults, representing 64.7 million people, suffered periodontitis, with $8.9 \%$ constituting severe cases (Eke et al., 2015). The occurrence of periodontitis is associated with sex/gender, race/ethnicity/skin color, education/schooling, socioeconomic classification/status/position, and income (Oppermann et al., 2015). In industrialized countries, oral health care based on private or public systems is available to the population, but many developing regions have few oral health services. Periodontitis is a burden on social and individual healthcare budgets (Petersen and Ogawa, 2005). According to the Korean National Health Insurance Service (NHIS), it is one of the most common oral diseases in Korea. The cost of health insurance for periodontitis represents a large and continuously growing proportion of NHIS expenditure (2013 Korean statistical annual report, http://www.nhis.or.kr). Thus, the need for risk factor-based periodontitis preventive strategies has increased.

Periodontitis is an inflammatory disease characterized by the host-mediated destruction of soft tissue caused by many factors, including stimulation of osteoclastogenesis (Marchbanks and Campbell, 1976). Various immune response genes are differentially expressed in this disease (Ohlrich et al., 2009). Moreover, it has recently been shown that polymorphisms of many of these, including single nucleotide polymorphisms (SNPS) in IL1B, IL6, TGF- $\beta 1$, and IL8, amongst others, are associated with chronic periodontitis (Ohlrich et al., 2009; Chen et al., 2014; Gabriela Teixeira et al., 2014; Huang et al., 2015; Lavu et al., 2015).

Caspases are intracellular proteases involved in apoptosis-, pyroptosis-, necroptosisand autophagy-mediated cell death (Los et al., 1999; Shalini et al., 2015). Many previous studies have elucidated the relationship between caspases and periodontitis. For instance, Aggregatibacter actinomycetemcomitans infection enhances apoptosis through a caspase 3-dependent mechanism (Kang et al., 2012). Furthermore, caspase 3 expression increases with progression of gingival inflammation, probing depth, and clinical attachment level in periodontitis (Pradeep et al., 2014), and tissue destruction by Porphyromonas gingivalis involves both caspase 1-dependent and -independent mechanisms (Taxman et al., 2006). Activation of caspases 3 and 7 is considerably enhanced in gingival tissue from periodontitis patients (Bantel et al., 2005), in whose osteoblasts the activation of caspase 8 has also been observed (Mori et al., 2009).

In the present study, we investigated whether the CASP gene polymorphisms are associated with periodontal disease risk in the Korean population. 


\section{MATERIAL AND METHODS}

\section{Participants}

This study was approved by the Institutional Review Board of the College of Dentistry, Dankook University, Cheonan, Republic of Korea (IRB No. H-1204/004/001). Written consent forms were obtained from all subjects. To investigate the association between CASP genes and chronic periodontitis susceptibility, 51 chronic periodontitis patients and 33 controls lacking the disease were recruited. Characteristics of the study groups are shown in Table 1. All individuals received oral and dental examinations. The periodontitis diagnosis criteria used are cited in Kang et al. (2015).

Table 1. Characteristics of the subjects included in this study.

\begin{tabular}{lcc}
\hline Characteristics & Controls & Periodontitis patients \\
\hline Number of subjects & 33 & 51 \\
Male & 24 & 25 \\
Female & 9 & 26 \\
Age (mean \pm SD) & $24.9 \pm 3.2$ & $49.68 \pm 9.9$ \\
\hline
\end{tabular}

$\mathrm{SD}=$ standard deviation.

\section{DNA extraction}

Genomic DNA was prepared from buccal cells. Before collecting cells from the inside of a patient's mouth, we confirmed the absence of any substance that might interfere with our DNA typing method by requiring patients to wash their mouths with water. Two sterilized swabs were used, one for each cheek, to collect cells from the buccal region. A swab was placed on the side of the cheek, and scraped across it over 10 times to obtain a sufficient quantity of epithelial cells. Each sample was extracted according to the DNA isolation kit manufacturer protocol (Roche, Mannheim, Germany).

\section{SNPs selection and genotyping}

We first searched for CASP genes containing SNPS (CASP1 to CASP10, CASP12, CASP14, and CASP8AP2) in the National Centre for Biotechnology Information (NCBI) database (http://www.ncbi.nlm.nih.gov/gene/). The selection criteria for exonic, promoter, and intronic SNPs in each gene were as follows: SNPs had to have 1) a minor allele frequency $>10 \%$; 2) heterozygosity $>0.1$; 3) known genotype frequencies in Asian populations; and 4) been examined in a previous study. We finally selected 201 CASP gene SNPs to investigate their association with chronic periodontitis (Table S1). Genotyping of all SNPs was carried out using an Axiom ${ }^{\mathrm{TM}}$ Genome-Wide Human Assay (Affymetrix, Inc., Santa Clara, CA, USA). All of the experimental procedures involved in this assay were performed at the Theragen Etex Corporation (Suwon, Republic of Korea) following the manufacturer protocol.

\section{Statistical analysis}

Hardy-Weinberg equilibrium (HWE) was assessed in both the patient and control groups using SNPStats (http://bioinfo.iconcologia.net/index.php?module=Snpstats). Genotype models (codominant, dominant, recessive, and log-additive) and logistic regression analysis were applied 
to determine whether differences in genotype frequencies between the periodontal disease and control groups were significant (Kim et al., 2013; Seok et al., 2014). SNPStats and SPSS 18.0 (SPSS, Inc., Chicago, IL, USA) were used for the statistical analysis. Logistic regression was conducted to evaluate odds ratios (ORs), 95\% confidence intervals $(95 \% \mathrm{Cls})$, and $P$ values. Linkage disequilibrium (LD) blocks and haplotypes were identified using Haploview version 4.2 (Barrett et al., 2005). For the statistical tests, the significance level for $P$ values was set at 0.05 .

\section{RESULTS}

A total of 201 CASP gene SNPs were analyzed (Table S2). Of these, only variants of CASP3 showed a significant association with periodontitis $(P<0.05)$. Table 2 shows the genotypic and allelic frequencies of these CASP3 polymorphisms in each study group [rs12108497, -894T>C; rs46476002, intron; and rs113420705, 5'-untranslated region (UTR)].

\begin{tabular}{|c|c|c|c|c|c|c|c|}
\hline SNP & Genotype or allele & Control [N (\%)] & Periodontitis [N (\%)] & Model & OR $(95 \% \mathrm{Cl})$ & $\mathrm{P}$ & Fisher exact $P$ \\
\hline CASP3 & $\mathrm{T} / \mathrm{T}$ & $18(60.0)$ & $13(25.5)$ & Codominant 1 & $3.52(1.30-9.56)$ & 0.013 & \\
\hline rs12108497 & $\mathrm{T} / \mathrm{C}$ & $11(36.7)$ & $28(54.9)$ & Codominant 2 & $13.85(1.57-121.94)$ & 0.018 & 0.006 \\
\hline \multirow[t]{5}{*}{$-894 T>C$} & $\mathrm{C} / \mathrm{C}$ & $1(3.3)$ & $10(19.6)$ & Dominant & $4.38(1.67-11.50)$ & 0.002 & \\
\hline & & & & Recessive & $7.07(0.86-58.31)$ & 0.024 & 0.05 \\
\hline & & & & Log additive & $3.61(1.61-8.12)$ & 0.0007 & \\
\hline & $\mathrm{T}$ & $47(78.3)$ & $54(52.9)$ & & 1 & & \\
\hline & C & $13(21.7)$ & $48(47.1)$ & & $3.21(1.55-6.65)$ & 0.002 & \\
\hline CASP3 & $T / T$ & $14(42.4)$ & $7(14.0)$ & Codominant 1 & $3.41(1.15-10.12)$ & 0.027 & \\
\hline rs4647602 & $T / G$ & $17(51.5)$ & $29(58.0)$ & Codominant 2 & $14.00(2.46-79.55)$ & 0.003 & 0.002 \\
\hline \multirow[t]{5}{*}{ Intron } & $\mathrm{G} / \mathrm{G}$ & $2(6.1)$ & $14(28.0)$ & Dominant & $4.53(1.57-13.01)$ & 0.0037 & \\
\hline & & & & Recessive & $6.03(1.27-28.61)$ & 0.0082 & 0.021 \\
\hline & & & & Log additive & $3.64(1.64-8.08)$ & 0.0005 & \\
\hline & $\mathrm{T}$ & $45(68.2)$ & $43(43.0)$ & & 1 & & \\
\hline & G & $21(31.8)$ & $57(57.0)$ & & $2.84(1.48-5.45)$ & 0.002 & \\
\hline CASP3 & $\mathrm{C} / \mathrm{C}$ & $16(50.0)$ & $10(19.6)$ & Codominant 1 & $3.52(1.30-9.55)$ & 0.013 & \\
\hline rs113420705 & $\mathrm{C} / \mathrm{T}$ & $15(46.9)$ & $33(64.7)$ & Codominant 2 & $12.80(1.38-118.32)$ & 0.025 & 0.018 \\
\hline \multirow[t]{5}{*}{ 5'-UTR } & $T / T$ & $1(3.1)$ & $8(15.7)$ & Dominant & $4.10(1.54-10.91)$ & 0.0038 & \\
\hline & & & & Recessive & $5.77(0.69-48.51)$ & 0.05 & 0.14 \\
\hline & & & & Log additive & $3.54(1.53-8.20)$ & 0.0015 & \\
\hline & $\mathrm{C}$ & $47(73.4)$ & $53(52.0)$ & & 1 & & \\
\hline & $\mathrm{T}$ & $17(26.6)$ & $49(48.0)$ & & $2.56(1.30-5.03)$ & 0.007 & \\
\hline
\end{tabular}

SNP = single nucleotide polymorphism; OR = odds ratio; $\mathrm{Cl}=$ confidence interval; UTR = untranslated region. Bold numbers indicate significant associations.

In the control group, the distribution of rs12108497 genotypes was in HWE $(P=1.00)$. Frequencies of $\mathrm{T} / \mathrm{T}, \mathrm{T} / \mathrm{C}$, and $\mathrm{C} / \mathrm{C}$ genotypes were $25.5,54.9$, and $19.6 \%$ in the periodontal disease group, and 60.0, 36.7, and $3.3 \%$ in the control group, respectively (Table 2). Logistic regression analyses for periodontitis patients and control subjects were carried out. Table 2 shows a significant difference in rs12108497 genotype frequencies $(P<0.05)$, and demonstrates that this SNP was significantly associated with periodontitis $(O R=3.52,95 \% \mathrm{Cl}=1.30-9.56, \mathrm{P}=0.013$ under codominant model $1 ; \mathrm{OR}=13.85,95 \% \mathrm{Cl}=1.57-121.94, \mathrm{P}=0.018$ under codominant model $2 ; \mathrm{OR}$ $=4.38,95 \% \mathrm{Cl}=1.67-11.50, \mathrm{P}=0.002$ under the dominant model $\mathrm{OR}=3.61,95 \% \mathrm{Cl}=1.61-8.12$, $\mathrm{P}=0.007$ under the log-additive model). The frequency of the rs $12108497 \mathrm{C}$ allele was higher in periodontitis patients $(47.1 \%)$ than in control subjects $(21.7 \%)$. Moreover, this difference was 
statistically significant $(\mathrm{OR}=3.21,95 \% \mathrm{Cl}=1.55-6.65, \mathrm{P}=0.002)$.

The distribution of rs4647602 genotypes in the control group was in HWE $(P=0.43)$. $\mathrm{T} / \mathrm{T}, \mathrm{T} / \mathrm{G}$, and $\mathrm{G} / \mathrm{G}$ genotype frequencies were $14.0,58.0$, and $28.0 \%$ in the periodontal disease group, and $42.4,51.5$, and $6.1 \%$ in the control group, respectively (Table 2). Logistic regression analyses for periodontitis patients and control subjects were carried out. Table 2 indicates that under all genotype models, rs4647602 showed significant $P$ values $(<0.05)$, revealing that this SNP was significantly associated with periodontitis $(\mathrm{OR}=3.41,95 \% \mathrm{Cl}=1.15-10.12, \mathrm{P}=0.027$ under codominant model $1 ; \mathrm{OR}=14.00,95 \% \mathrm{Cl}=2.46-79.55, \mathrm{P}=0.003$ under codominant model $2 ; \mathrm{OR}$ $=4.53,95 \% \mathrm{Cl}=1.57-13.01, \mathrm{P}=0.0037$ under the dominant model; $\mathrm{OR}=6.03,95 \% \mathrm{Cl}=1.27$ $28.61, \mathrm{P}=0.0082$ under the recessive model; $\mathrm{OR}=3.64,95 \% \mathrm{Cl}=1.64-8.08, \mathrm{P}=0.005$ under the log-additive model). The frequency of the rs4647602 $\mathrm{G}$ allele was higher in the periodontitis group $(57.0 \%)$ than in the controls $(31.8 \%)$, and this difference was found to be statistically significant $(\mathrm{OR}=2.84,95 \% \mathrm{Cl}=1.48-5.45, \mathrm{P}=0.002)$.

In the control group, the distribution of rs113420705 genotypes was in HWE $(P=0.43)$.

The frequencies of $\mathrm{C} / \mathrm{C}, \mathrm{C} / \mathrm{T}$, and $\mathrm{T} / \mathrm{T}$ genotypes were $19.6,64.7$, and $15.7 \%$ in the periodontitis group, and $50.0,46.9$, and $3.1 \%$ in the control group, respectively (Table 2). Logistic regression analyses for each study group were carried out. In Table 2, it can be seen that under each genotype model except for recessive model, the rs113420705 SNP showed significant $P$ values $(<0.05)$, demonstrating an association with periodontitis $(\mathrm{OR}=3.52,95 \% \mathrm{Cl}=1.30-9.55, \mathrm{P}=0.013$ under codominant model $1 ; \mathrm{OR}=12.80,95 \% \mathrm{Cl}=1.38-118.32, \mathrm{P}=0.025$ under codominant model $2 ; \mathrm{OR}$ $=4.10,95 \% \mathrm{Cl}=1.54-10.91, \mathrm{P}=0.0038$ under the dominant model $\mathrm{OR}=3.54,95 \% \mathrm{Cl}=1.53-8.20$, $\mathrm{P}=0.0015$ under the log-additive model). The rs113420705 $\mathrm{T}$ allele frequency was higher in the periodontitis group (48.0\%) than in the control group (26.6\%), and this difference was statistically significant $(\mathrm{OR}=2.56,95 \% \mathrm{Cl}=1.30-5.03, \mathrm{P}=0.007)$.

These results indicate that the C, G, and T alleles of rs12108497, rs4647602, and rs113420705, respectively, may constitute risk factors for the development of periodontitis.

In order to further investigate the relationship between these three CASP3 SNPS and periodontitis, the presence of LD blocks between rs12108497, rs4647602, and rs113420705 was assessed using Haploview version 4.2. One LD block was constructed (rs4647602 and rs113420705; $D^{\prime}=0.968, r^{2}=0.69 ;$ Figure 1), defining three haplotypes. Of these haplotypes, two (TC and GT) were found to be associated with periodontitis (TC, P =0.0039; GT, P = 0.002; Table 3).

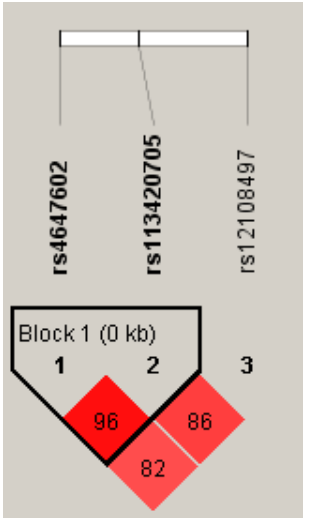

Figure 1. Linkage disequilibrium block defined by the CASP3 single nucleotide polymorphisms tested. 
Table 3. Haplotype analysis of CASP3 polymorphisms in control and periodontal disease groups.

\begin{tabular}{|c|c|c|c|c|c|c|c|}
\hline \multirow[t]{2}{*}{ Haplotype } & \multirow[t]{2}{*}{ Frequency } & \multicolumn{2}{|c|}{ Control } & \multicolumn{2}{|c|}{ Periodontitis } & \multirow[t]{2}{*}{ Chi-square } & \multirow[t]{2}{*}{$P$} \\
\hline & & + & - & + & - & & \\
\hline TC & 0.527 & 43.9 & 22.1 & 44.7 & 57.3 & 8.347 & 0.0039 \\
\hline GT & 0.386 & 16.0 & 50.0 & 48.9 & 53.1 & 9.558 & 0.002 \\
\hline GC & 0.080 & 5.0 & 61.0 & 8.3 & 93.7 & 0.016 & 0.8991 \\
\hline
\end{tabular}

Bold numbers indicate significant associations. The haplotype consisted of single nucleotide polymorphisms rs 4647602 and rs113420705.

\section{DISCUSSION}

Chronic periodontitis could represent a persistent reservoir of microparticles released by multiple cells in an inflammatory context, and hence might be associated with increased prevalence of systemic diseases (Badran et al., 2015). The link between periodontitis and diabetes mellitus is well known (Grossi and Genco, 1998). Furthermore, the prevalence and incidence of coronary heart disease (Bahekar et al., 2007) and the risk of atherosclerotic cardiovascular disease (Dietrich et al., 2013) is increased in periodontitis. Periodontal treatment reduces the risk of cardiovascular disease by lowering plasma levels of inflammatory and thrombotic markers and adhesion molecules (Saffiet al., 2015). Periodontitis is related to rheumatoid arthritis, and treatment for the former has an effect on both the latter and systemic inflammation (Payne et al., 2015). In addition, preeclampsia (Ha et al., 2014), other complications during pregnancy (Zi et al., 2015), and inflammatory bowel disease (Vavricka et al., 2013), amongst other conditions, have been shown to be associated with chronic periodontitis.

This disease is significantly linked to socioeconomic influences, such as low income, low education level, socioenvironmental factors, race, and ethnic group (Petersen and Ogawa, 2005). Indeed, it is a more prevalent and severe problem in developing countries (Pilot, 1998). In the US, periodontitis prevalence is highest in Hispanics (63.5\%) and non-Hispanic blacks (59.1\%), followed by non-Hispanic Asian Americans (50.0\%), and is lowest in non-Hispanic whites (40.8\%; Ekeet al., 2015). In groups of similar socioeconomic status, access to social security and possession of health insurance has been shown to be important risk factors for periodontitis (Singh et al., 2014). This disease is a major public health problem worldwide and effective prevention methods are needed.

Periodontitis risk is influenced by many factors, and several reports have shown that genetic characteristics make an important contribution. A study of self-reported periodontal disease in a Virginia twin population showed the key role of genetic factors for adult-onset periodontal disease risk (Corey et al., 1993), and oral morphology and function have been observed to be influenced by genetic components (Kurushima et al., 2015). Furthermore, data from twin studies indicate that both genetic and environmental circumstances affect periodontal disease (Michalowicz, 1994). Recently, many investigations have reported associations between individual genetic polymorphisms and chronic periodontitis susceptibility, involving many genes including IL1B, IL1RN, TNF- $\alpha$, TGF- $\beta 1$, IL8, CCL5, COX2, DEFB1, MMP1, MMP9, STAT1, CD14, IKKI, LT$\alpha$, MiR146a, MiR499, OPG, RANKL, and VDR.

In this study, we investigated CASP genes in the NCBI database containing SNPs (CASP1 to CASP10, CASP12, CASP14, and CASP8AP2). Caspases are proteolytic enzymes known for their role in controlling cell death and inflammation. However, their functions extend beyond this to include cell proliferation, tumor suppression, differentiation, neural development, and axon guidance and aging (Shalini al., 2015). Our results revealed a relationship between CASP3 
SNPs and periodontitis. A number of previous enquiries have established an association between caspases and chronic periodontitis but none have reported a link between this condition and CASP gene polymorphism. For example, the concentration of caspase 3 in gingival crevicular fluid and serum has been seen to proportionally increase with the progression of periodontal disease (Abuhussein et al., 2014; Pradeep et al., 2014). Moreover, Aggregatibacter actinomycetemcomitans infection enhances apoptosis of osteoblastic cells, reducing osseous coupling through a caspase 3-dependent mechanism (Kang et al., 2012; Pacios et al., 2013). Caspase 3 is also involved in the Porphyromonas gingivalis-derived lipopolysaccharide-mediated activation of the MAPK signaling pathway in human periodontal ligament fibroblasts (Seo et al., 2012). In addition to its role in apoptosis, caspase 3 is crucial for bone formation and the differentiation of bone marrow stromal stem cells into osteoblasts, due to its influence on the TGF- $\beta / S M A D 2$ pathway and cell cycle progression (Miura et al., 2004).

In the current work, the C, G, and T alleles of rs12108497, rs4647602, and rs 113420705, respectively, were found to be associated with the development of periodontitis. rs 12108497 has previously been linked to stomach and gastric cancers, while rs4647602 is associated with squamous cell carcinoma of the head and neck and atrial fibrillation, and rs113420705 is involved in coronary artery lesion formation in Kawasaki disease. Periodontitis leads to inflammatory responses in the atrial myocardium, disturbing the structural properties and electrophysiology of the atrium, facilitating its fibrillation (Yu et al., 2010). Moreover, periodontal bone loss is associated with complex multiple coronary lesions (Romagna et al., 2012). Altogether, these results might provide further evidence for the role of caspase 3 in chronic periodontitis events, such as cell apoptosis, inflammation, and bone loss.

The prevalence of edentulousness in the elderly is low in many industrialized countries but remains high in developing regions. Periodontitis and tooth loss are linked to quality of life and chronic diseases, and thus constitute a global burden. Early diagnosis utilizing genetic factors and prevention of chronic periodontitis could improve oral health and reduce social and individual healthcare costs.

To conclude, CASP3 gene polymorphisms may be markers of susceptibility to periodontal disease in the Korean population. Additional studies with larger sample sizes and a focus on different populations will need to be conducted to confirm our results.

\section{Conflicts of interest}

The authors declare no conflict of interest.

\section{ACKNOWLEDGMENTS}

Research supported by the Basic Science Research Program through the National Research Foundation of Korea (NRF) funded by the Ministry of Science, ICT and Future Planning (\#NRF-2011-0013194).

\section{Supplementary material}

\section{REFERENCES}

Abuhussein H, Bashutski JD, Dabiri D, Halubai S, et al. (2014). The role of factors associated with apoptosis in assessing 
periodontal disease status. J. Periodontol. 85: 1086-1095.

Badran Z, Struillou X, Verner C, Clee T, et al. (2015). Periodontitis as a risk factor for systemic disease: Are microparticles the missing link? Med. Hypotheses 84: 555-556.

Bahekar AA, Singh S, Saha S, Molnar J, et al. (2007). The prevalence and incidence of coronary heart disease is significantly increased in periodontitis: a meta-analysis. Am. Heart J. 154: 830-837.

Bantel H, Beikler T, Flemmig TF and Schulze-Osthoff K (2005). Caspase activation is involved in chronic periodontitis. FEBS Lett. 579: 5559-5564.

Barrett JC, Fry B, Maller J and Daly MJ (2005). Haploview: analysis and visualization of LD and haplotype maps. Bioinformatics 15: 263-265.

Burt BA, Ismail AI, Morrison EC and Beltran ED (1990). Risk factors for tooth loss over a 28-year period. J. Dent. Res. 69: 1126-1130

Chen X, Huang J, Zhong L and Ding C (2015). Quantitative assessment of the associations between interleukin-8 polymorphisms and periodontitis susceptibility. J. Periodontol. 86: 292-300.

Corey LA, Nance WE, Hofstede P and Schenkein HA (1993). Self-reported periodontal disease in a Virginia twin population. J. Periodontol. 64: 1205-1208.

Dietrich T, Sharma P, Walter C, Weston P, et al. (2013). The epidemiological evidence behind the association between periodontitis and incident atherosclerotic cardiovascular disease. J. Periodontol. 84: S70-S84.

Eke PI, Dye BA, Wei L, Slade GD, et al. (2015). Update on Prevalence of Periodontitis in Adults in the United States: NHANES 2009 to 2012. J. Periodontol. 86: 611-622.

Gabriela Teixeira F, Mendonca SA, Menezes Oliveira K, Barbosa Dos Santos D, et al. (2014). Interleukin-6 c.-174G>C Polymorphism and Periodontitis in a Brazilian Population. Mol. Biol. Int. 2014: 490308.

Gerritsen AE, Allen PF, Witter DJ, Bronkhorst EM, et al. (2010). Tooth loss and oral health-related quality of life: a systematic review and meta-analysis. Health Qual. Life Outcomes 8: 126.

Grossi SG and Genco RJ (1998). Periodontal disease and diabetes mellitus: a two-way relationship. Ann. Periodontol. 3: 51-61.

Ha JE, Jun JK, Ko HJ, Paik DI, et al. (2014). Association between periodontitis and preeclampsia in never-smokers: a prospective study. J. Clin. Periodontol. 41: 869-874.

Huang J, Ding C, Chen X, He R, et al. (2015). Association of TGF- $\beta 1-509 C / T$, +869T/C, and +915G/C polymorphisms with periodontitis susceptibility. Oral Dis. 21: 443-450.

Kang J, de Brito Bezerra B, Pacios S, Andriankaja O, et al. (2012). Aggregatibacter actinomycetemcomitans infection enhances apoptosis in vivo through a caspase-3-dependent mechanism in experimental periodontitis. Infect. Immun. 80: 2247-2256.

Kang SW, Han SY, Lim SB, Cho KB, et al. (2015). ACE insertion/deletion polymorphism is associated with periodontal disease in Korean population. Arch. Oral Biol. 60: 496-500.

Kim H-K, Lee HY and Kim H-J (2013). A polymorphism in DMT1 is associated with lead-related hypertensive status. Mol. Cell Toxicol. 9: 415-420.

Kurushima Y, Ikebe K, Matsuda K, Enoki K, et al. (2015). Influence of genetic and environmental factors on oral diseases and function in aged twins. J. Oral Rehabil. 42: 49-56.

Lavu V, Venkatesan V, Venkata Kameswara Subrahmanya Lakkakula B, Venugopal P, et al. (2015). Polymorphic regions in the interleukin-1 gene and susceptibility to chronic periodontitis: agenetic association study. Genet. Test. Mol. Biomarkers 19: 175-181.

Los M, Wesselborg S and Schulze-Osthoff K (1999). The role of caspases in development, immunity, and apoptotic signal transduction: lessons from knockout mice. Immunity 10: 629-639.

Marchbanks RM and Campbell CW (1976). Sodium and chloride fluxes in synaptosomes in vitro. J. Neurochem. 26: 973-980.

Michalowicz BS (1994). Genetic and heritable risk factors in periodontal disease. J. Periodontol. 65: 479-488.

Miura M, Chen XD, Allen MR, Bi Y, et al. (2004). A crucial role of caspase-3 in osteogenic differentiation of bone marrow stromal stem cells. J. Clin. Invest. 114: 1704-1713.

Mori G, Brunetti G, Colucci S, Oranger A, et al. (2009). Osteoblast apoptosis in periodontal disease: role of TNF-related apoptosis-inducing ligand. Int. J. Immunopathol. Pharmacol. 22: 95-103.

Ohlrich EJ, Cullinan MP and Seymour GJ (2009). The immunopathogenesis of periodontal disease. Aust. Dent. J. 54 (Suppl 1): S2-S10.

Oppermann RV, Haas AN, Rosing CK and Susin C (2015). Epidemiology of periodontal diseases in adults from Latin America. Periodontol. 2000 67: 13-33.

Pacios S, Andriankaja O, Kang J, Alnammary M, et al. (2013). Bacterial infection increases periodontal bone loss in diabetic rats through enhanced apoptosis. Am. J. Pathol. 183: 1928-1935.

Payne JB, Golub LM, Thiele GM and Mikuls TR (2015). The Link Between Periodontitis and Rheumatoid Arthritis: A Periodontist's Perspective. Curr. Oral Health Rep. 2: 20-29.

Petersen PE (2003). The World Oral Health Report 2003: continuous improvement of oral health in the 21st century - the 
approach of the WHO Global Oral Health Programme. Community Dent. Oral Epidemiol. 31 (Suppl 1): 3-23.

Petersen PE and Ogawa $\mathrm{H}$ (2005). Strengthening the prevention of periodontal disease: the WHO approach. J. Periodontol. 76: 2187-2193.

Pilot T (1998). The periodontal disease problem. A comparison between industrialised and developing countries. Int. Dent. J. 48: 221-232.

Pradeep AR, Suke DK, Prasad MV, Singh SP, et al. (2014). Expression of key executioner of apoptosis caspase-3 in periodontal health and disease. J. Investig. Clin. Dent. 5: 1-6.

Romagna C, Dufour L, Troisgros O, Lorgis L, et al. (2012). Periodontal disease: a new factor associated with the presence of multiple complex coronary lesions. J. Clin. Periodontol. 39: 38-44.

Saffi MA, Furtado MV, Polanczyk CA, Montenegro MM, et al. (2015). Relationship between vascular endothelium and periodontal disease in atherosclerotic lesions: Review article. World J. Cardiol. 7: 26-30.

Seo T, Cha S, Kim TI, Lee JS, et al. (2012). Porphyromonas gingivalis-derived lipopolysaccharide-mediated activation of MAPK signaling regulates inflammatory response and differentiation in human periodontal ligament fibroblasts. J. Microbiol. 50: 311-319.

Seok H, Kim SK, Yoo KH, Lee BC, et al. (2014). Association of BID SNPs (rs8190315 and rs2072392) and clinical features of benign prostate hyperplasia in Korean population. J. Exerc. Rehabil. 10: 383-388.

Shalini S, Dorstyn L, Dawar S and Kumar S (2015). Old, new and emerging functions of caspases. Cell Death Differ. 22: 526-539.

Singh A, Purohit BM, Masih N and Kahndelwal PK (2014). Risk factors for oral diseases among workers with and without dental insurance in a national social security scheme in India. Int. Dent. J. 64: 89-95.

Taxman DJ, Zhang J, Champagne C, Bergstralh DT, et al. (2006). Cutting edge: ASC mediates the induction of multiple cytokines by Porphyromonas gingivalis via caspase-1-dependent and -independent pathways. J. Immunol. 177: 4252-4256.

Vavricka SR, Manser CN, Hediger S, Vogelin M, et al. (2013). Periodontitis and gingivitis in inflammatory bowel disease: a case-control study. Inflamm. Bowel Dis. 19: 2768-2777.

Yu G, Yu Y, Li YN and Shu R (2010). Effect of periodontitis on susceptibility to atrial fibrillation in an animal model. J. Electrocardiol. 43: 359-366.

Zi MY, Longo PL, Bueno-Silva B and Mayer MP (2015). Mechanisms involved in the association between periodontitis and complications in pregnancy. Front. Public Health 2: 290. 